\title{
Diagnóstico de bienestar animal en un criadero intensivo de carpinchos (Hydrochoerus hydrochaeris) en Salto, Uruguay
}

\author{
Mahia Elizabeth Minteguiaga-Boutureira*, Lic., MSc. (c), \\ ${ }_{1}$ Estudiante de posgrado, Facultad de Medicina Veterinaria, Universidad de la República, Montevideo, Uruguay
}

Recibido: 21 de mayo del 2015 Aprobado: 12 de diciembre del 2015

*Autor de correspondencia: Mahia Minteguiaga Boutureira. Facultad de Química, Universidad de la República. Montevideo, Uruguay. Teléfono: 094047 492. Correo electrónico: mahiaminte@gmail.com

Cómo citar este artículo: Minteguiaga-Boutureira ME. Diagnóstico de bienestar animal en un criadero intensivo de carpinchos (Hydrochoerus hydrochaeris) en Salto, Uruguay. Spei Domus. 2015;11(23):47-54. doi: http://dx.doi.org/10.16925/ sp.v11i23.1367

Resumen. Propósito: en el marco de la elaboración de una tesis de maestría en comportamiento reproductivo (programa de posgrado de la Facultad de Veterinaria, Universidad de la República, Uruguay), se procedió a evaluar el nivel de bienestar animal del plantel de carpinchos (H. hydrochaeris) de un criadero de sistema intensivo de producción. Temas: la cría del carpincho en Uruguay aún no se ha afianzado, pese a que el consumo ilegal de su carne y el uso de ejemplares como mascotas es una realidad innegable. Pero las pocas iniciativas de cría legal de la especie no han tenido mayor éxito. Desarrollo: para la evaluación del nivel de bienestar, se observó el comportamiento de los animales (mediante registros sistemáticos y observaciones casuales) y se consultó al productor sobre el mantenimiento y el manejo de los animales del criadero. Más allá de algunos problemas puntuales (disponibilidad de agua, disponibilidad diferencial de alimentos), los ejemplares son mantenidos en un nivel aceptable de bienestar. Conclusiones: esta iniciativa privada a pequeña escala mantiene a sus ejemplares en condiciones de bienestar animal aceptables, a pesar del escaso interés por parte de las autoridades (de diversos ministerios) de fomentar iniciativas de este tipo, como forma de frenar la caza ilegal y proteger las poblaciones silvestres.

Palabras clave: bienestar animal, fauna silvestre, Hydrochoerus hydrochaeris, recurso zoogenético. 


\title{
Diagnosis of Animal Welfare at an Intensive Capybara (Hydrochoerus hydrochaeris) Breeding Center In Salto, Uruguay
}

\begin{abstract}
Purpose: As part of the preparation of a master's thesis in reproductive behavior (graduate program of the School of Veterinary Medicine, Universidad de la República, Uruguay), the level of animal welfare of capybara (H. hydrochaeris) stock at a breeding center with an intensive production system was assessed. Topics: Capybara breeding in Uruguay has not yet been consolidated, although the illegal consumption of its meat and use of some specimens as pets is an undeniable reality. However, the few initiatives for legal breeding of the species have not been successful. Development: To assess the level of welfare, animal behavior was observed (by systematic records and casual observations) and the producer was asked about maintenance and management of animals at the breeding center. Beyond some specific problems (water availability, differential food availability), specimens are maintained at an acceptable level of welfare. Conclusions: This private smallscale undertaking keeps its specimens in acceptable conditions of animal welfare, despite the lack of interest by the authorities (from various ministries) in promoting such initiatives as a way to curb poaching and protect wild populations.
\end{abstract}

Keywords: animal welfare, wildlife, Hydrochoerus hydrochaeris, animal genetic resource.

\section{Diagnóstico de bem-estar numa instalação animal de criação intensiva de capivaras (Hydrochoerus hydrochaeris) no Salto, Uruguay}

Resumo. Propósito: no âmbito da elaboração de uma dissertação de mestrado em comportamento reprodutivo (programa de pós-graduação da Faculdade de Veterinária da Universidade da República, Uruguai), procedeu-se a avaliar o nível de bem-estar animal das capivaras (H. hydrochaeris) de um criatório num sistema intensivo de produção. Temas: a criação do capivara no Uruguai ainda não está consolidada, embora o consumo ilegal de sua carne e o uso de exemplares como animais de estimação seja uma realidade inegável. Contudo, as poucas iniciativas de criação legal da espécie não vêm tendo sucesso. Desenvolvimento: para a avaliação do nível de bem-estar, observou-se o comportamento dos animais (mediante registros sistemáticos e observações casuais) e consultou-se o produtor sobre a manutenção e a gestão dos animais do criadouro. Mais além de alguns problemas pontuais (disponibilidade de água e de diferentes alimentos), os exemplares são mantidos num nível aceitável de bem-estar. Conclusões: essa iniciativa privada a pequena escala mantém seus exemplares em condições de bem-estar animal aceitáveis, apesar do escasso interesse das autoridades (de diversos ministérios) de promover iniciativas desse tipo, como forma de frear a caça ilegal e proteger as populações silvestres.

Palavras-chave: bem-estar animal, fauna silvestre, Hydrochoerus hydrochaeris, recurso zoogenético. 


\section{Introducción}

\section{El carpincho como especie de producción alternativa}

El carpincho, capibara o chigüire (Hydrochoerus hydrochaeris) ha sido utilizado como fuente natural de proteínas en la alimentación de los pueblos sudamericanos. Se trata del roedor viviente más grande del mundo actual, preciado por la calidad de su carne, la fineza de su cuero y las propiedades de su grasa [1, $2,3]$. Su distribución geográfica se extiende por todos los países desde Panamá hasta el sur de Argentina, con excepción de Chile.

Al ser el carpincho un herbívoro primario que se alimenta sobre todo de gramíneas y plantas acuáticas, puede adaptarse fácilmente a otros tipos de alimentos como descartes de producción hortícola y ración comercial balanceada. El abrigo, ya sea en forma de sombras artificiales, vegetación o refugios de diversos tipos, es fundamental para esta especie en cautiverio. Uno de los usos de este recurso es como lugar de parición. La disponibilidad de cuerpos de agua de tamaño diverso es muy importante para la cópula, la termorregulación, beber y esconderse de predadores $[4,5]$.

En Uruguay, existen algunos criaderos autorizados por Renare (Recursos Naturales Renovables del Ministerio de Ganadería, Agricultura y Pesca-MGaP [6]), que deben cumplir con disposiciones comunes a criaderos de fauna silvestre y con reglamentaciones específicas para esta especie. De todas formas, las poblaciones silvestres de carpincho han disminuido debido a la caza indiscriminada y a la destrucción de su hábitat [7]. Sumado al hecho de que se trata de una explotación no tradicional, los criadores se enfrentan a la falta de conocimiento de la biología reproductiva y social de la especie, sobre todo de sus aspectos de conducta. El mantenimiento de animales silvestres en cautiverio, ya sea en zoológicos o para su explotación comercial, conlleva modificaciones en su ambiente y su conducta que pueden provocar problemas en su bienestar [8].

\section{Sistemas de cría del carpincho}

Hay tres sistemas de explotación del carpincho: extensivo, semicautiverio e intensivo. El sistema extensivo se emplea sobre todo en grandes territorios dedicados exclusivamente a varias familias de carpinchos.
El país donde más se desarrolla esta práctica es en Venezuela, en la zona de los Llanos [9]. Las investigaciones a finales de la década de 1960, elaboradas por Ojasti, determinaron que en dichas poblaciones silvestres con una carga de 0,6 animales / ha se puede extraer hasta un $30 \%$ de la población al año sin afectar la estabilidad demográfica de la especie [10].

Este sistema utiliza el mínimo manejo humano posible, y deja a las poblaciones desarrollar su vida normalmente hasta que llega la época de sequía y la consiguiente matanza. Los grupos se reúnen cerca de los cuerpos de agua que subsisten y allí se procede en una forma poco humanitaria a un abate a garrotazos [11]. La tradición venezolana implica el consumo de la carne del "chiguire" conservada en sal para las Pascuas, tal cual se consume pescado en las mismas fechas en otras regiones, por lo que luego de la faena se lo procesa de esa forma.

El sistema con más aceptación y rentabilidad es el de cría en semicautiverio. En este método, pueden criarse las familias de carpinchos a partir de parejas de reproductores adquiridas a otros productores o zoológicos, o utilizar animales que ya se encuentren de forma natural en el predio (estancias, campos). Se establecen "piquetes" o "potreros" de 0,5 a 0,6 ha para grupos de un macho dominante y siete hembras, o de 1 ha para 15 hembras y dos machos. Cada potrero debe contener uno o varios cuerpos de agua, refugio y sombra, ya sean naturales o artificiales. Se hace un manejo mínimo (sexado, separación de juveniles para conformar nuevos grupos y faena), para lo cual se pueden utilizar mangas o tubos similares a los utilizados para ganados bovinos con adaptaciones a esta especie. Se recomienda la faena al primer año de edad (juvenil), al alcanzar un peso de 35 a $45 \mathrm{~kg}$. Los animales son cebados con alimento sólido de su preferencia hacia las mangas, y luego de capturados son llevados a los frigoríficos. Allí se procede a un abate humanitario, con insensibilización previa por electronarcosis y posterior sangría [12].

En la cría intensiva, los animales son confinados en recintos de dimensiones reducidas, lo que implica mayorcontrolymanejo,mayorproductividadydemejor calidad, y mejores condiciones sanitarias [7]. Debido al comportamiento social de estos animales y a su alto grado de territorialidad, la conformación y disposición de los grupos en los recintos se vuelve un factor clave en el éxito del emprendimiento productivo. Se proponen $40 \mathrm{~m}^{2}$ /animal, con grupos de un macho dominante y hasta ocho hembras por recinto [12]. Este sistema 
es más demandante en cuanto a mano de obra y es menos rentable si se lo considera como una explotación independiente. Al constante aporte de pastura fresca, se le puede sumar la ración sólida, la limpieza de los tanques de agua y el mantenimiento de los cercos que dividen los recintos y refugios. El proceso de faena es similar al planteado para el sistema de cría de semicautiverio.

\section{Objetivo}

Evaluar el nivel de bienestar animal en carpinchos de un establecimiento de cría intensiva en Uruguay.

\section{Materiales y métodos}

\section{El criadero "Don Javier"}

El espacio físico del criadero "Don Javier" constaba de ocho encierros que se fueron modificando estructuralmente durante el periodo de estudio, totalizando unos $1500 \mathrm{~m}^{2}$ de instalaciones. Estaban construidos con tejido de alambre, postes de madera y chapas metálicas. Todos contaban con un refugio, árboles de sombra, bateas o recipientes para disponer la ración, y áreas de baño. El criadero está en una chacra, por lo que se dispone de descarte de la producción hortícola (zanahorias, boniatos y zapallos, entre otros) y de forraje natural para alimentar a los ejemplares, además de ración balanceada brindada por el productor. El régimen de alimentación es de dos veces al día, todos los días: temprano en la mañana y a última hora del día.

Se trata de un pequeño emprendimiento privado, casi de características artesanales, propiedad del Sr. Javier Marzaroli, que comenzó sus actividades en el 2001 tras cumplir con los requerimientos legales del Departamento de Fauna del MGAP de Uruguay. Una vez contactado el productor en el 2009 para conseguir su permiso para la realización de este estudio a largo plazo, se acordó su plena participación y su autorización para difundir las actividades de su criadero.

Todos los animales que participaron en el estudio nacieron en cautiverio en el criadero. El plantel del establecimiento se mantuvo en cerca de 30 animales. Para los objetivos de la tesis, se observó sistemáticamente el comportamiento reproductivo de machos y hembras del plantel, y las interacciones entre madres y sus crías hasta que cumplían los 3 meses de vida. Pero las condiciones de mantenimiento y el comportamiento general de todos los ejemplares del criadero se observaron en paralelo, de manera anecdótica.

\section{Evaluación}

Con base en observaciones del comportamiento del plantel y de las instalaciones del criadero "Don Javier" ( $31^{\circ} 27^{\prime} 36,82^{\prime \prime}$ S 58 $50^{\circ} 00^{\prime} 34,32^{\prime \prime}$ O, departamento de Salto, Uruguay), se elaboró el siguiente reporte sobre las condiciones de bienestar de estos animales. La evaluación utilizó el protocolo de Welfare Quality [13]. Estas medidas se evaluaron a nivel individual o de grupo en una escala de tres niveles $(0,1$ y 2$)$, criterio establecido por la autora. Una puntuación de 0 equivale a un bienestar satisfactorio, una puntuación de 1 es cuando el bienestar animal está comprometido y una puntuación de 2 se da en caso de que el bienestar sea pobre e inaceptable.

\section{Resultados}

En la tabla 1, se sintetiza el resultado de la evaluación.

\section{Alimentación}

Ausencia de hambre prolongada: calificación $=1$. Los animales son alimentados con pastura natural y ración balanceada comercial (afrechillo, semitín) todos los días, más la provisión temporaria de descartes de producción hortícola (zanahoria, boniato). La pastura y los vegetales se depositan esparcidos sobre el piso del encierro, mientras que la ración se deposita en bateas. El régimen de alimentación es de dos veces al día, todos los días: temprano en la mañana y a última hora del día.

Problema 1: la alimentación de hembras gestantes y en lactancia, así como en crías destetadas; es en estos periodos cuando la calidad y la cantidad del alimento deben variar para satisfacer las necesidades energéticas de los animales. Esto no se tiene en cuenta en este criadero. Problema 2: el productor comentó que en los periodos de sequía o inundaciones se le hace muy difícil proporcionarle pastura a los animales. 
Tabla 1. Evaluación de bienestar en un criadero de carpinchos (H. hydrochaeris) en Uruguay, según los criterios de Welfare Quality

\begin{tabular}{|l|l|c|l|}
\hline \multicolumn{1}{|c|}{ Principios } & \multicolumn{1}{|c|}{ Criterios } & \multicolumn{1}{c|}{ Resultado } & \multicolumn{1}{c|}{ Categorización } \\
\hline \multirow{4}{*}{ Alimentación } & Ausencia de hambre prolongada & 1 & Comprometido \\
\cline { 2 - 4 } & Ausencia de sed prolongada & 0 & Aceptable \\
\hline \multirow{5}{*}{ Alojamiento } & Confort en relación con el descanso & 0 & Aceptable \\
\cline { 2 - 4 } & Confort térmico & 1 & Comprometido \\
\cline { 2 - 4 } & Facilidad de movimiento & 0 & Aceptable \\
\hline \multirow{5}{*}{ Comportamiento animal sanitario } & Ausencia de lesiones & 0 & Aceptable \\
\cline { 2 - 4 } & Ausencia de enfermedades & 2 & Pobre \\
\cline { 2 - 4 } & Ausencia de dolor causado por el manejo & 0 & Aceptable \\
\cline { 2 - 4 } & Expresión de comportamiento social adecuado & 0 & Aceptable \\
\cline { 2 - 4 } & Expresión adecuada de otras conductas & 0 & Aceptable \\
\cline { 2 - 4 } & Relación humano-animal positiva & 0 & Aceptable \\
\cline { 2 - 4 } & Estado emocional positivo & 0 & 0 \\
\hline
\end{tabular}

Fuente: elaboración propia

Ausencia de sed prolongada: calificación $=0$. Todos los encierros cuentan con algún tipo de cuerpo de agua de donde los animales pueden beber, que son lo suficientemente grandes para evitar encuentros agonísticos entre los individuos. El predio cuenta con pozo de agua surgente y bomba. Debido a la costumbre de excretar en el agua con la que se bañan y de la que beben, el recambio se hace dos veces por semana en el verano y una vez por semana en invierno. En uno de los encierros se construyó una piscina de concreto, mientras que en otro se tiene un pequeño estanque natural y en los demás se utilizan tanques de metal.

Problema: en estos mismos cuerpos de agua (naturales, tanques o piscinas de concreto) los animales suelen defecar y orinar, y son utilizados para baños (termorregulación) y comportamientos sexuales (cópula). El productor no cuenta con un sistema de flujo de agua constante sino que cambia el agua y limpia los tanques y piscinas semanalmente. Esto puede acarrear enfermedades, sobre todo en meses cálidos cuando la materia fecal se descompone más rápidamente.

\section{Alojamiento}

Conforten relación con el descanso: calificación $=0$. Las áreas externas de los recintos son lo suficientemente amplias para el descanso de todos los animales. La superficie dentro de los refugios y en el exterior es de tierra y pasto. Cuentan con áreas de defecación perfectamente separadas de las de descanso

Confort térmico: calificación $=1$. Problema: a pesar de ser animales resistentes, los carpinchos necesitan abrigo y sombra. Los refugios son insuficientes para albergar a más de cuatro individuos, y hay grupos de hasta diez animales. Algunos recintos no producen la sombra suficiente en horas pico de sol y los cuerpos de agua no son lo suficientemente amplios para todos los animales.

Facilidad de movimiento: calificación $=0$. Los animales están sueltos en un área adecuada, de acuerdo con las recomendaciones de otros autores. Se estima un área por animal aproximada de $32 \mathrm{~m} 2$ en este criadero.

\section{Estado sanitario}

Ausencia de lesiones: calificación $=0$. Las lesiones presentadas son producto de interacciones sociales normales entre los individuos, sobre todo entre los grupos en los que no se han separado los machos o las introducciones de nuevos animales no son graduales. Hay relaciones de dominancia entre los machos de esta especie bien definidas [14], pero no así entre las hembras, en las que se observaron 
amenazas a la hora del acceso a bateas con ración o pasturas. Estos animales tienen una capacidad de cicatrización formidable, por lo cual no implicaría un problema para su salud.

Ausencia de enfermedades: calificación $=2$. Problema: se han dado varias muertes tanto de adultos como de juveniles. Se presenta una diarrea, sin síntomas previos de decaimiento o falta de apetito, para terminar a las pocas horas con la muerte del animal. Ante la escasa información de los veterinarios consultados y sin poder establecer las causas primeras de la enfermedad, no se puede aplicar ningún tratamiento preventivo. Tampoco hay suministros de vacunas ni otro tipo de manejo sanitario (desparasitación, controles de rutina, detección de preñez, etc.).

Ausencia de dolor causado por el manejo: calificación $=0$. No se hace ningún tipo de manejo rutinario que consista en manipular a los animales. $\mathrm{Si}$ se los tiene que cambiar de un recinto a otro, se los arrea con vocalizaciones normales. Las crías se sexan manualmente, en un procedimiento de duración breve y manipulación cuidadosa.

\section{Comportamiento}

Expresión de comportamiento social adecuado: calificación $=0$. Los animales son mantenidos en grupos con un solo macho, a fin de evitar conductas agonísticas con los machos subordinados. Las crías son separadas de sus madres a los casi 5 meses, ya concluido el amamantamiento, para formar los nuevos grupos reproductivos. Problema: se dan algunos comportamientos agonísticos entre grupos reproductivos adyacentes. Al ser animales muy territoriales y de conformación social cerrada, la proximidad de otros animales adultos extraños genera agresividad. También, se han escapado crías muy pequeñas por entre los orificios del alambrado hacia recintos de adultos o hacia el exterior.

Expresión adecuada de otras conductas: calificación $=0$. Los animales exploran su recinto, los adultos marcan con sus glándulas sobre distintos sustratos. El comportamiento sexual no se ve afectado por el cautiverio, pues aunque en vida libre la cópula se realiza en el agua, han sabido adaptarse a los cuerpos de agua presentes o simplemente copulan en tierra, dada la ausencia de predadores. No hay comportamientos anormales ni estereotipias en ninguno de los animales del plantel.
Relación humano-animal positiva: calificación $=0$. Durante el manejo rutinario (alimentación, limpieza de tanques/piscinas), los animales se alejan de forma moderada o se acercan con curiosidad.

Estado emocional positivo: calificación $=0$. Las crías y los juveniles juegan entre ellos y con los adultos. Los adultos retozan en el agua cuando tienen espacio suficiente.

Para esta evaluación, se utilizaron indicadores comportamentales, dado que son la base de la investigación de la tesis, pero sin llegar a cuantificarlos, pues no es el fin del trabajo. Se podrían utilizar indicadores de producción, como ganancia de peso, e indicadores fisiológicos. En estos casos, se debería hacer una medición indirecta, pues aunque el manejo sea mínimo y estén acostumbrados a la presencia del operario/productor, los carpinchos tienden a estresarse frente a situaciones nuevas, lo que podría afectar los valores de lo que queremos medir (cortisol en sangre por ejemplo). Tampoco están validados los valores de esta hormona para saliva o fecas, por lo que se debería optar por otros indicadores como frecuencia cardíaca o temperatura. En un reciente trabajo de Nogueira et al. [15], se plantea el uso de las vocalizaciones ultrasónicas como un posible indicador comportamental de bienestar en esta especie.

\section{Conclusiones}

Las condiciones de cautiverio intensivo del criadero "Don Javier" (Salto, Uruguay) no traen problemas mayores al bienestar animal de los carpinchos. Con algunas modificaciones en la infraestructura y el manejo, sobre todo en lo sanitario, los problemas encontrados pueden solucionarse.

\section{Perspectivas}

Algunas recomendaciones puntuales a los problemas planteados en la evaluación de bienestar serían:

- El productor debería contar con reservas de pasturas para periodos climáticos críticos, así como reforzar la alimentación de las hembras y crías con suplementos alimenticios apropiados.

- Se debería contar con bebederos apropiados para evitar la ingesta de agua contaminada o, 
en su defecto, instalar un sistema de flujo continuo de agua en los tanques y en la piscina. Si esto tampoco fuera posible, el productor debería cambiar el agua con más frecuencia; dos veces por semana en meses fríos y cada dos días en meses cálidos.

- Es imperioso reconstruir los refugios actuales que resultan inapropiados para alojar a los grupos en épocas de estrés térmico. Se debe proporcionar sombra artificial en aquellos recintos en los que la vegetación existente no brinda la sombra necesaria. Los cuerpos de agua deben ser del tamaño apropiado según los animales que contenga el recinto. En la mayoría de ellos se debería aumentar su tamaño.

- Se debe contar con un médico veterinario con la formación apropiada para la atención de estos animales, así sea para asesorar a algún profesional local en cuanto al manejo sanitario.

- Se debe disponer de varios recintos en buenas condiciones que impidan el pasaje de animales de un grupo a otro, así como ataques entre las vallas. Se recomienda una disposición que intercale grupos reproductivos y grupos de juveniles, y reforzar la malla de recintos con crías.

El principal escollo de esta explotación en Uruguay, según comenta el productor, radica en el momento de la faena y la venta. Actualmente, no hay ningún convenio con frigoríficos para el faenado habilitado de las carcasas, y por lo tanto, la carne no puede ser comercializada. El productor tiene que encargarse del sacrificio si llega algún comprador, utilizando una escopeta, y la faena la realiza el comprador clandestinamente fuera del criadero para evitarle problemas al productor.

La escasez del apoyo del Ministerio de Ganadería y del Instituto de Carnes a esta iniciativa de producción alternativa está desalentando a los productores, que no consiguen la rentabilidad suficiente como para apostar a mejorar sus emprendimientos y a lograr mayor alcance con sus productos. De esta forma, lo que podría ser una iniciativa positiva que ofreciera un producto de buena calidad y que desalentara la caza furtiva en vida libre del carpincho pareciera estar al borde del naufragio a falta de buena voluntad de las autoridades estatales.

\section{Nota}

Este artículo es producto de la participación del autor en el curso virtual sobre Bienestar en Animales de Producción, realizado por la Protección Animal Mundial (WAP, por sus siglas en ingles) en el 2014.

La toma de datos se llevó a cabo durante el desarrollo de la tesis de maestría de la autora en Reproducción Animal, titulada "Descripción de cortejo, cópula e interacción madre-cría en carpincho (H. hydrochaeris), en condiciones de cría intensiva", bajo la tutoría del Dr. Daniel Olazábal. Contó con el financiamiento de la Agencia Nacional de Investigación e Innovación del Uruguay (Beca Pos_ 2011_1_3483).

Los resultados se expusieron en dos instancias: 1) v Jornadas Uruguayas de Comportamiento Animal, Montevideo, 2015. "Huele a romance: la importancia del marcaje en la reproducción del carpincho ( $H$. hydrochaeris) en cautiverio", de Minteguiaga y Olazábal; 2) II Congreso Latinoamericano de Etología Aplicada, Ilheus, Brasil, 2011. "Descripción de cortejo y cópula en tierra, en cautiverio intensivo, entre carpinchos (Hydrochoerus hydrochaeris) no familiarizados entre sî", de Minteguiaga y Olazábal.

$\mathrm{Al}$ momento de la tesis, la reglamentación del programa de posgrado de la Facultad de Veterinaria de la Universidad de la República no estipulaba ninguna autorización de los Comités de Ética. De todas formas, para el otorgamiento de la beca de la Agencia Nacional de Investigación e Innovación (ANII), se necesita que el estudiante esté habilitado por la Comisión Honoraria de Experimentación Animal de la Universidad de la República, y la autora tiene la habilitación en la categoría в de dicha comisión.

\section{Referencias}

[1] Oda SHI, Bressan MC, Fonseca de Freitas RT, Miguel GZ, Vieira JO, Faria PB, Savian TV. Composição centesimal e teor de colesterol dos cortes comerciais de capivara (Hydrochaeris hydrochaeris L. 1766). Ciênc Agrotec Lavras. 2004;6(1):1344-51. 
[2] Bressan MC, Oda SH, Cardoso MG, Miguel GZ, Fonseca de Freitas RT, Vieira JO, Faria PB, Savian TV Ferrão SPB. Composição de ácidos graxos dos cortes comerciais de capivara (Hydrochaeris hydrochaeris L. 1766). Ciênc Agrotec Lavras, 2004:6(1):1352-59.

[3] Saadoun A, Cabrera MC. A review of the nutritional content and technological parameters of indigenous sources of meat in South America. Meat Sci. 2008;80(1):570-81.

[4] Alho CJR. Criação e manejo de capivaras em pequenas propriedades rurais. Brasilia: Embrapa; 1986

[5] Mones A, Ojasti J. Hydrochoerus hydrochaeris. Mammalian Species. 1986;264(1):1-7.

[6] Renare, Ministerio de Ganadería, Agricultura y Pesca (MGAP). Decreto 186/002, Instalación de criaderos de especies animales de la fauna silvestre en régimen de cautiverio (23 mayo 2002).

[7] Álvarez MR, Kravetz F. Propuesta para el aprovechamiento sustentable del carpincho (Hydrochoerus hydrochaeris, Rodentia) en Argentina. En: Memorias vi Congreso Internacional sobre Manejo de Fauna Silvestre en la Amazonia y Latinoamérica. Lima; 2004. p. $405-14$

[8] Hötzel MJ, Nogueira SSC, Pinheiro Machado Filho LC. Bem-estar de animais de produção: das necessidades animais às possibilidades humanas. Revista de Etologia. 2010;9(2):1-10.
[9] Giraldo D, Ramírez J. Guía para el manejo, cría y aprovechamiento sostenible del chigüiro, chigüire o capibara Hydrochoerus hydrochaeris Linneo. Bogotá: Convenio Andrés Bello.

[10] Mayor Aparicio P, Santos Fita D, López Béjar M. La cría de capibara (Hydrochoerus hydrochaeris). En: Sostenibilidad de la Amazonía y la cría de animales silvestres. Lima: Centro de Estudios Teológicos de la Amazonía; 2007. p. 98-109.

[11] Herrera EA. The effect of harvesting on the age structure and body size of a capybara population. Ecotropicos. 1992;5(1):20-5.

[12] Pinheiro MS, Centeno Da Silva JJ, Rodrigues RC. Sistemas de criação de capivaras. Brasil. Embrapa Clima Temperado Documentos N. ${ }^{\circ}$ 152; 2005

[13] Welfare Quality [Internet]. Disponible en: www.welfarequality.net

[14] Herrera EA. Capybara social behavior and use of space: patterns and processes. En: JR Moreira et al., editores. Capybara: biology, use and conservation of an exceptional neotropical species. Nueva York: Springer Science + Business Media; 2013. p. 195-207.

[15] [15] Nogueira SSC, Barros KS, Almeida MH, Pedroza JP, Nogueira Filho SLG, Tokumaru RS. Ultrasound call detection in capybara. Pesquisa Veterinária Brasileira. 2012;32(7):663-6. 